\title{
Corrigendum: Emerging Vibrio risk at high latitudes in response to ocean warming
}

Craig Baker-Austin, Joaquin A. Trinanes, Nick G. H. Taylor, Rachel Hartnell, Anja Siitonen and Jaime Martinez-Urtaza

Nature Climate Change 3, 73-77 (2013); published online 22 July 2012; corrected after print 27 June 2016

In the version of this Letter originally published, an outdated version of Figure panel $3 \mathrm{~d}$ was displayed in Fig. 3, and so the population density and risk data shown was incorrect. Figure panel $3 \mathrm{~d}$ has been replaced in the online version of this Letter. 